\title{
INSAR ERROR BUDGET FOR LARGE SCALE DEFORMATION
}

\author{
F. De Zan, A. Parizzi, F. Rodriguez Gonzalez, H. Ansari, G. Gomba, R. Brcic, M. Eineder
}

German Aerospace Center (DLR), Oberpfaffenhofen, Germany

\begin{abstract}
The capacity of SAR interferometry to measure surface deformation with accuracy of $1 \mathrm{~mm} /$ year or better are well known. However this is typically limited to relative motion at short distance. Thanks to several advances in SAR sensor quality, data availability, orbit determination, processing, and calibration of atmospheric delays it is now possible to achieve that accuracy even across large distances of hundreds of kilometers.

In this paper we revise the main contributions to the large scale error, considering available mitigation techniques. We provide a first validation for a processing based on Sentinel-1 data, by comparing our results with GNSS stations.

For future SAR's operating at lower frequencies, it is vital to consider ionospheric corrections and likely also the influence of moisture variations in natural scatterers. The choice of processing algorithms, though typically not discussed, can also have a significant effect on the quality of the result.
\end{abstract}

Index Terms - SAR interferometry, large scale deformation, troposphere, ionosphere, soil moisture, space geodesy

\section{INTRODUCTION}

SAR Interferometry (InSAR) is today well established in measuring fast local deformations in the centimeter/decimeter range, e.g. of volcanoes and of earthquakes, or local deformation rates with high accuracy (e.g. $1 \mathrm{~mm} / \mathrm{year}$ ). Unfortunately, the signals before an earthquake when strain slowly accumulates are distributed over wide areas (e.g. $300 \mathrm{~km}$ ). Measuring this type of large-scale small signals is still the domain of permanent ground-based GNSS networks but this is now changing thanks to recent advancements in the processing and calibration methods.

The contributions to the interferometric phase that we routinely observe in the interferograms are many: geometry/topography, surface deformation, propagation through ionosphere and troposphere etc. A typical problem of SAR interferometry is the separation and mitigation of unwanted contributions to allow a proper interpretation and improved quality of the measurement.

In this paper we are going to revisit quantitatively the contributions and corrections, especially in relation to large scale deformation monitoring. Some contributions are relevant at all frequencies, others are especially relevant to lower frequency SAR's, like many of the upcoming missions (SAOCOM, NISAR, BIOMASS, Tandem-L).

We will first review the phase error budget and the typical contributions to the range error; then we will hint at contributions that are not completely understood yet, but are nonetheless relevant. Performance models have a tendency to concentrate on well characterized parts of reality, forgetting other aspects.

\section{PERFORMANCE ANALYSIS FOR DEFORMATION RATE}

Typically the strength of InSAR stays with measuring nearby points, differential motion at short distances. For example in C-band, for two nearby point targets with a SNR just above 1 , the relative motion can be determined with an accuracy of a few millimeter and the deformation rate with accuracy easily smaller than $1 \mathrm{~mm} / \mathrm{y}$ with regular Sentinel-1 stacks. Large scale processes have been until now out of reach, as there measurements were contaminated by many other sources, beside thermal/clutter noise. These sources, like atmospheric delays or limited orbit knowledge, have a low-pass character, which means that the errors at large distances are much larger than at short distances. Nowadays, thanks to many technical advancements, the goal of determining relative deformation with an accuracy of $1 \mathrm{~mm} / \mathrm{year}$ at large distances (100's of $\mathrm{km}$ ) has become a realistic one.

Let's therefore focus on the estimation of a linear velocity, which is essentially a line fit. The performance for a regular acquisition scenario is approximately:

$$
\sigma_{v}=\frac{\sqrt{2} \sigma_{r} / \sqrt{N / 3}}{2 T / 3}
$$

where $\sigma_{r}$ is the range error on a single measurements (single SAR acquisition, between two points), $N$ is the number of images, and $T$ the total time span. Let's assume that our SAR stack spans $T=8$ years, with 50 acquisitions per year. It is immediate to verify that to reach an accuracy of $\sigma_{v}=1 \mathrm{~mm} / \mathrm{y}$, the total range error on each image must be in the order of $3 \mathrm{~cm}$. This analysis assumes of course uncorrelated errors; if the error exhibits a trend over the time span, the performance will be worse. We will now analyze several contributions to 
the range error $\sigma_{r}$, to show that a "1 $\mathrm{mm} / \mathrm{y}$ " performance is achievable even across large distances.

\section{INSTRUMENT AND GEOMETRY}

A first contribution is the orbit knowledge. An orbit error in elevation (in the zero Doppler plane, orthogonal to the line of sight) translates in a phase ramp across the swath. With typical figures for LEO satellites, an error in the across-track plane of $5 \mathrm{~cm}$ corresponds to a range ramp of $1.5 \mathrm{~cm}$. Such orbit errors are reasonable for current SAR satellites [1] and additional errors from the antenna patterns are typically negligible. For future satellites, based on reflectors, the knowledge of the antenna patterns and their variations will need to introduce errors of comparable magnitude.

In the past, long-term drift and jitter in the oscillator frequency have produced phase trends from near to far range, that could be mistaken for genuine motion (ERS \& ENVISAT). Considering the performance target of $1 \mathrm{~mm} /$ year across the swath, the oscillator should be stable enough to avoid drifts of $1 \mathrm{~mm} / 100 \mathrm{~km} /$ year $=10^{-8} /$ year. The figure $100 \mathrm{~km}$ represents roughly the slant range that corresponds to a ground range of $300 \mathrm{~km}$, i.e. a typical swath of future spaceborne SAR's. Fortunately, with Sentinel-1, the drifts are smaller than $10^{-9} /$ year [2] and they can be ignored. One must say that if the oscillator drift can be well modeled, the magnitude of the drift is not decisive but its knowledge is then the crucial point.

Generally speaking, modern satellites have very good navigation capabilities (both in knowledge and control). This, together with good digital elevation models, allows precise correction of the geometric phase. The difficulty resides more in the interpretation / understanding of the measurement, rather than in the measurements itself, as it is the case for the azimuth-varying line of sight in the ScanSAR and TOPS modes.

\section{ATMOSPHERIC EFFECTS}

\subsection{Troposphere}

Tropospheric delays are caused by variations of temperature, pressure and humidity of the earth atmosphere. The variable fraction amounts to a few centimeters. Variations in the delays can be related both to changes in the meteorological parameters and to changes in the observation geometry, like incidence angle variations in the swath and topography-related effects.

According to our experience, once numerical weather prediction models are used to correct the tropospheric delay, the absolute residual variation is in the order of $0.5-3$ centimeters [3] depending on the geographical location, or even less, using the latest ECMWF products like ERA-5. Because of the limited resolution of the ECMWF models $(30 \mathrm{~km}$, ERA5) it will be difficult to catch and compensate the turbulent part of the troposphere. Given the fractal spectrum of the troposphere delay, these corrections are particularly relevant for InSAR measurements on large scale, for example $100 \mathrm{~km}$ or larger, or for sites characterized by large topographic variations where the stratified component of the troposphere plays a big role. The improvement at large scales compared to the case with no corrections is a factor 10 in the error power, which corresponds to about a factor 3 in standard deviation.

The residual error can be accommodated within the range error requirement derived above $(3 \mathrm{~cm})$ or exceeds it only slightly, depending on the geographical location. Notice that the tropospheric delay is not dependent on the radar wavelength when it is expressed in units of length.
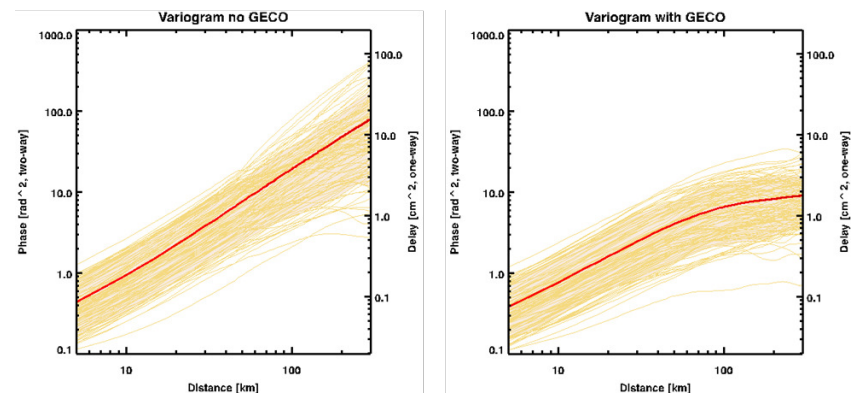

Fig. 1. Variograms of the interferometric phases before (left) and after (right) the corrections. The single variograms are depicted in yellow; the mean behavior in red.

\subsection{Ionosphere}

Ionospheric range delays are instead very sensitive to the frequency. Their magnitude is proportional to the square of the wavelength, so that longer wavelengths are much more affected. Many ALOS/PALSAR and ALOS-2/PALSAR-2 examples show that an operational use of L-band data requires routine correction of ionospheric effects. In our experience we found it much more reliable exploiting the dispersion of the SAR signal (split spectrum technique) than relying on external GNSS-based models for the corrections [4]. The ionospheric range delay can be reduced down to residuals of $1 \mathrm{~cm}$ for an area of $1 \mathrm{~km}^{2}$ [5], starting from several meter delay in L-band. In most situation this is enough and fits within the range error budget. Fast spatial variations of the ionosphere require more sophisticated techniques.

The ionosphere affects naturally longer wavelength more than shorter, however we have shown that many C-band data are affected. Moreover, the performance of split-spectrum corrections depends only on the range bandwidth and not on the central frequency. A particular problem that affects ScanSAR or TOPS acquisition modes is that the ionosphere is not sampled continuously and particular care has to be taken 
when smoothing the estimated ionospheric delay across different bursts. We have successfully applied the split-spectrum corrections to Sentinel-1 IW data (C-band) [4].

\section{MOISTURE AND OTHER EFFECTS}

Propagation in semi-transparent media, like many natural targets, is affected by the moisture status. Expected variable contributions to the delay are $2-4 \mathrm{~cm}$ for a typical soil type when observed in L-band [6]. A model developed by some of the authors predicts a magnitude proportional to the wavelength, which means that these effects are more relevant in Lband than in C-band [7]. Apart from the estimation of moisture itself [8], we are investigating the possibility to further mitigate its impact on the deformation measurements. A difficulty with phase errors driven by moisture is that there might well be long-term trends that impact the deformation rate estimation much more significantly than random errors. This disturbance might turn out to be a performance bottleneck when attempting to reconstruct surface deformations with high accuracy over large scales.

A similar effect is given by the propagation through snow. Whereas the dielectric constant of dry snow depends only on the density, for wet snow it is necessary to consider also the frequency dependency.

\section{PROCESSING}

Processing plays an important albeit often neglected role in keeping the quality of InSAR measurements. When dealing with distributed scatterers it is common to apply spatial filtering and use a large set of interferograms, supposedly redundant, typically selecting them on the basis of the coherence or spatio-temporal separation. However any method based on interferogram stacking relies, often implicitly, on an integration step of temporal differences and is therefore prone to integration errors in the form of drifts.

This is especially dangerous when the interferometric phases present a significant non-closure [9], something that can be easily observed over natural scatterers, for instance because of moisture variations. The integration error can easily reach several $\mathrm{cm} /$ year in the estimated rates. Careful interferometric processing seems to partially protect from this kind of errors and keep the budget [10]. A safe alternative could be using only permanent/persistent scatterers, avoiding any spatial averaging. Depending on the implementation even these methods might suffer from integration errors, but rather in the spatial domain.

\section{EXAMPLE}

Figure 2 shows one example of large-scale processing with 3.4 years of Sentinel-1 data. The covered scene is about 600 $\mathrm{km}$ long and $250 \mathrm{~km}$ wide. The phases have been corrected

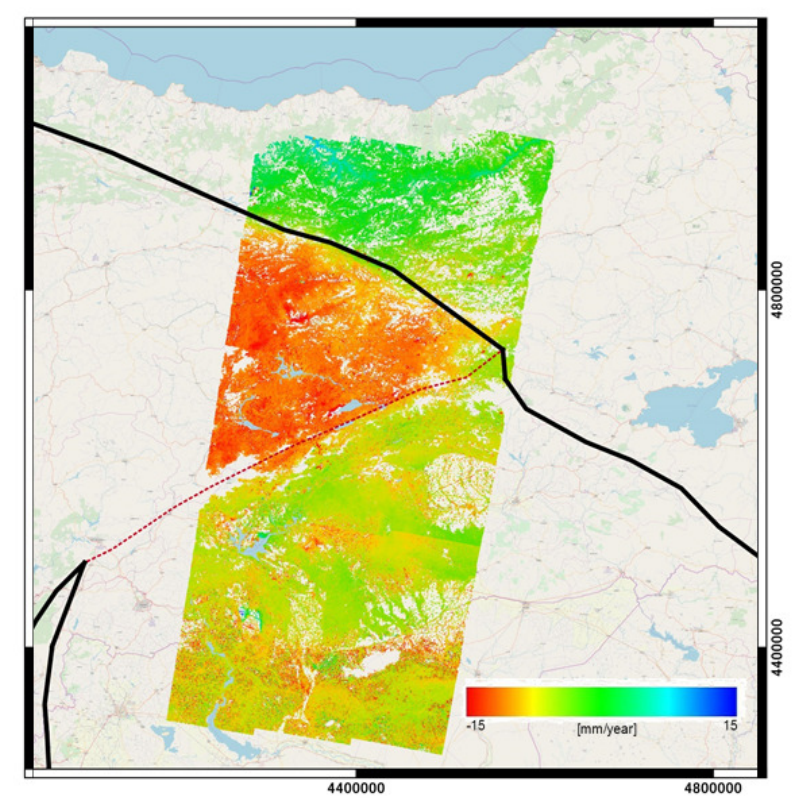

Fig. 2. Map of LoS deformation rate (mm/year) of North and East Anatolian faults.

for the ionospheric and tropospheric components, and Earth tides; the final product has been referred to the Eurasian plate. The results were calibrated estimating a single offset w.r.t. a set of 15 GPS station from a global GPS network (Nevada Geodetic Laboratory, [11]), a step that might even prove unnecessary in the future.

The errors with respect to the GPS network are about $1 \mathrm{~mm} /$ year in standard deviation, to be scaled by $\sqrt{2}$ to represent the error between two uncorrelated points, which is therefore $1.4 \mathrm{~mm} /$ year. To confirm this rather low figure we can consider the variograms of the residual phase screen of interferometric pairs with short temporal baselines (Figure 1) which saturate for large distances at about $2 \mathrm{~cm}^{2}$ for two acquisitions, or $1 \mathrm{~cm}^{2}$ for a single acquisition. This should include all atmospheric residuals plus orbital effects and would predict an error in the deformation rate of about $1 \mathrm{~mm} / \mathrm{year}$. One must say that part of the observed error of $1.4 \mathrm{~mm} / \mathrm{year}$ might come from the comparison with GNSS. This demonstration confirms the growing capability of InSAR processing in retrieving large scale tectonic motion. Notice that elsewhere in the world the tropospheric corrections will have a lower performance and the $\mathrm{mm} /$ year threshold will require longer time spans to be achieved, e.g. 8 years instead of 3 4 years. 


\section{CONCLUSIONS}

Advances in sensor quality, orbit determination, processing, and calibration allow millimeter/year accuracy over large scales with a few years of acquisition. This is particularly true for permanent/persistent scatterer techniques and we have given an example with Sentinel-1 data with a validation against GNSS. When distributed scatterers are included in the analysis, the effects related to moisture variations and error in the temporal integration have to be carefully mitigated.

\section{REFERENCES}

[1] H. Peter, A. Jäggi, J. Fernández, D. Escobar, F. Ayuga, D. Arnold, M. Wermuth, S. Hackel, M. Otten, W. Simons, P. Visser, U. Hugentobler, and P. Féménias, "Sentinel-1A first precise orbit determination results," Advances in Space Research, vol. 60, no. 5, pp. 879 892, 2017.

[2] Y. Larsen, P. Marinkovic, J. F. Dehls, Z. Perski, A. J. Hooper, and T. J. Wright, "The Sentinel-1 constellation for InSAR applications: Experiences from the InSARAP project," in 2017 IEEE International Geoscience and Remote Sensing Symposium (IGARSS), 2017, pp. 5545-5548.

[3] X. Cong, U. Balss, F. Rodriguez Gonzalez, and M. Eineder, "Mitigation of tropospheric delay in SAR and InSAR using NWP data: Its validation and application examples," Remote Sensing, vol. 10, no. 10, 2018.

[4] G. Gomba, F. Rodríguez González, and F. De Zan, "Ionospheric phase screen compensation for the Sentinel-1 TOPS and ALOS-2 ScanSAR modes," IEEE Transactions on Geoscience and Remote Sensing, vol. 55, no. 1, pp. 223-235, 2017.

[5] G. Gomba, A. Parizzi, F. De Zan, M. Eineder, and R. Bamler, "Toward operational compensation of ionospheric effects in sar interferograms: The split-spectrum method," IEEE Transactions on Geoscience and Remote Sensing, vol. 54, no. 3, pp. 1446-1461, March 2016.

[6] S. Zwieback, S. Hensley, and I. Hajnsek, "Assessment of soil moisture effects on L-band radar interferometry," Remote Sensing of Environment, vol. 164, pp. 77-89, 2015 .

[7] F. De Zan, A. Parizzi, P. Prats-Iraola, and P. LópezDekker, "A SAR interferometric model for soil moisture," IEEE Transactions on Geoscience and Remote Sensing, vol. 52, no. 1, pp. 418-425, Jan 2014.

[8] F. De Zan and G. Gomba, "Vegetation and soil moisture inversion from SAR closure phases: First experiments and results," Remote Sensing of Environment, vol. 217, pp. $562-572,2018$.

[9] F. De Zan, M. Zonno, and P. López-Dekker, "Phase inconsistencies and multiple scattering in SAR interferometry," IEEE Transactions on Geoscience and Remote Sensing, vol. 53, no. 12, pp. 6608-6616, Dec 2015.

[10] H. Ansari, F. De Zan, and R. Bamler, "Sequential estimator: Toward efficient InSAR time series analysis," IEEE Transactions on Geoscience and Remote Sensing, vol. 55, no. 10, pp. 5637-5652, 2017.

[11] G. Blewitt, W. C. Hammond, and C. Kreemer, "Harnessing the GPS data explosion for interdisciplinary science," Eos, vol. 99, 2018. 